Network Working Group

Request for Comments: 2384

Category: Standards Track
R. Gellens

QUALCOMM, Incorporated

August 1998

POP URL Scheme

Status of this Memo

This document specifies an Internet standards track protocol for the Internet community, and requests discussion and suggestions for improvements. Please refer to the current edition of the "Internet Official Protocol standards" (STD 1) for the standardization state and status of this protocol. Distribution of this memo is unlimited.

Copyright Notice

Copyright (C) The Internet Society (1998). All Rights Reserved.

1. Introduction

[POP3] is a widely-deployed mail access protocol. Many programs access POP3 message stores, and thus need POP3 configuration

information. Since there are multiple configuration elements which are required in order to access a mailbox, a single string representation is convenient.

A POP3 mailbox (like an [IMAP4] mailbox) is a network resource, and URLs are a widely-supported generalized representation of network resources.

A means of specifying a POP3 mailbox as a URL will likely be useful in many programs and protocols. [ACAP] is one case where a string encapsulation of elements required to access network services is needed. For example, an [IMAP4] message store is usually specified in ACAP datasets as an [IMAP-URL].

This memo defines a URL scheme for referencing a POP mailbox.

2. Conventions Used in this Document

The key words "MUST", "MUST NOT", "SHOULD", "SHOULD NOT", and "MAY" in this document are to be interpreted as defined in "Key words for use in RFCs to Indicate Requirement Levels" [KEYWORDS]. 


\section{POP Scheme}

The POP URL scheme designates a POP server, and optionally a port number, authentication mechanism, authentication ID, and/or authorization ID.

The POP URL follows the common Internet scheme syntax as defined in RFC 1738 [BASIC-URL] except that clear text passwords are not permitted. If : pport> is omitted, the port defaults to 110 .

The POP URL is described using [ABNF] in section 8.

A POP URL is of the general form:

pop: / / <user $>$; auth $=<$ auth $>$ e $<$ host $>$ : $<$ port $>$

Where <user>, <host>, and <port> are as defined in RFC 1738, and some or all of the elements, except "pop://" and <host>, may be omitted.

4. POP User Name and Authentication Mechanism

An authorization (which mailbox to access) and authentication (whose password to check against) identity (referred to as "user name" for simplicity) and/or authentication mechanism name may be supplied. These are used in a "USER", "APOP", "AUTH" [POP-AUTH], or extension command after making the connection to the POP server. If the URL doesn't supply an authentication identifier, the program interpreting the POP URL SHOULD request one from the user.

An authentication mechanism can be expressed by adding "; AUTH=<encauth-type>" to the end of the user name. If the authentication mechanism name is not preceded by a "+", it is a SASL POP [SASL] mechanism. If it is preceded by a "+", it is either "APOP" or an extension mechanism.

When an <enc-auth-type> is specified, the client SHould request appropriate credentials from that mechanism and use the "AUTH", "APOP", or extension command instead of the "USER" command. If no user name is specified, one SHOULD be obtained from the mechanism or requested from the user as appropriate.

The string "; AUTH=*" indicates that the client SHOULD select an appropriate authentication mechanism. It MAY use any mechanism supported by the POP server.

If an <enc-auth-type> other than ";AUTH=*" is specified, the client SHOULD NOT use a different mechanism without explicit user permission. 
If a user name is included with no authentication mechanism, then

"; $\mathrm{AUTH}=\star "$ is assumed.

Since URLs can easily come from untrusted sources, care must be taken when resolving a URL which requires or requests any sort of authentication. If authentication credentials are supplied to the wrong server, it may compromise the security of the user's account. The program resolving the URL should make sure it meets at least one of the following criteria in this case:

(1) The URL comes from a trusted source, such as a referral server which the client has validated and trusts according to site policy. Note that user entry of the URL may or may not count as a trusted source, depending on the experience level of the user and site policy.

(2) Explicit local site policy permits the client to connect to the server in the URL. For example, if the client knows the site domain name, site policy may dictate that any hostname ending in that domain is trusted.

(3) The user confirms that connecting to that domain name with the specified credentials and/or mechanism is permitted.

(4) A mechanism is used which validates the server before passing potentially compromising client credentials.

(5) An authentication mechanism is used which will not reveal information to the server which could be used to compromise future connections.

A URL containing "; AUTH=*" should be treated with extra care since it might fall back on a weaker security mechanism. Finally, clients are discouraged from using a plain text password as a fallback with

"; $\mathrm{AUTH}=*$ " unless the connection has strong encryption (e.g., a key length of greater than 56 bits).

Note that if unsafe or reserved characters such as " " or "; " are present in the user name or authentication mechanism, they MUST be encoded as described in RFC 1738 [BASIC-URL].

5. Relative POP URLs

Relative POP URLs are not permitted. 
6. Multinational Considerations

Since 8-bit characters are not permitted in URLs, [UTF8] characters are encoded as required by the URL specification [BASIC-URL] .

\section{Examples}

The following examples demonstrate how a POP client program might translate various POP URLs into a series of POP commands. Commands sent from the client to the server are prefixed with "C:", and responses sent from the server to the client are prefixed with "S:".

The URL:

<pop://rg@mailsrv.qualcomm.com>

Results in the following client commands:

<request password from user>

<connect to mailsrv.qualcomm.com, port 110>

S: +OK POP3 server ready <1896.697170952@mailsrv.qualcomm.com>

C: USER rg

$S:+O K$

$C:$ PASS secret

S: +OK rg's mailbox has 2 messages (320 octets)

The URL:

<pop: //rg; AUTH=+APOP@mail.eudora.com:8110>

Results in the following client commands:

<client requests password from user>

<connect to mail.eudora.com, port 8110>

$\mathrm{S}$ : +OK POP3 server ready <1896.697170952@mail.eudora.com>

C: APOP rg c4c9334bac560ecc979e58001b3e22fb

$\mathrm{S}:$ +OK mailbox has 1 message (369 octets)

The URL:

<pop: / /baz; AUTH=SCRAM-MD5efoo.bar >

Results in the following client commands:

<connect to foo.bar, port 110>

S: +OK POP3 server ready <1896.697170952@foo.bar $>$

C: AUTH SCRAM-MD5 AGNocmlzADx0NG40UGFiOUhCMEFtL1FMWEI3MmVnQGVsZW 


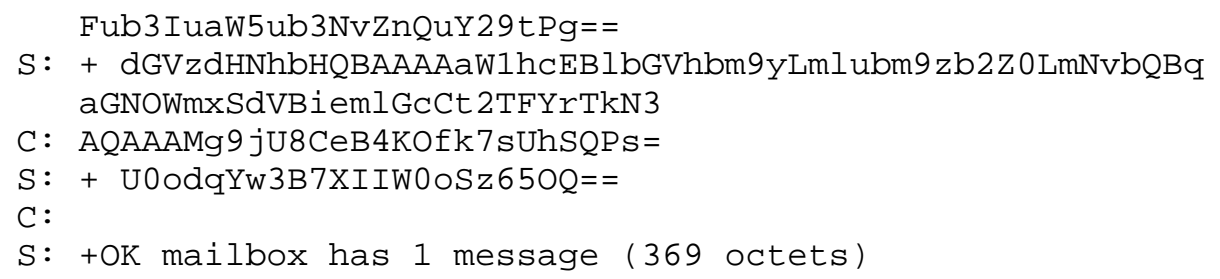

8. ABNF for POP URL scheme

The POP URL scheme is described using [ABNF]:

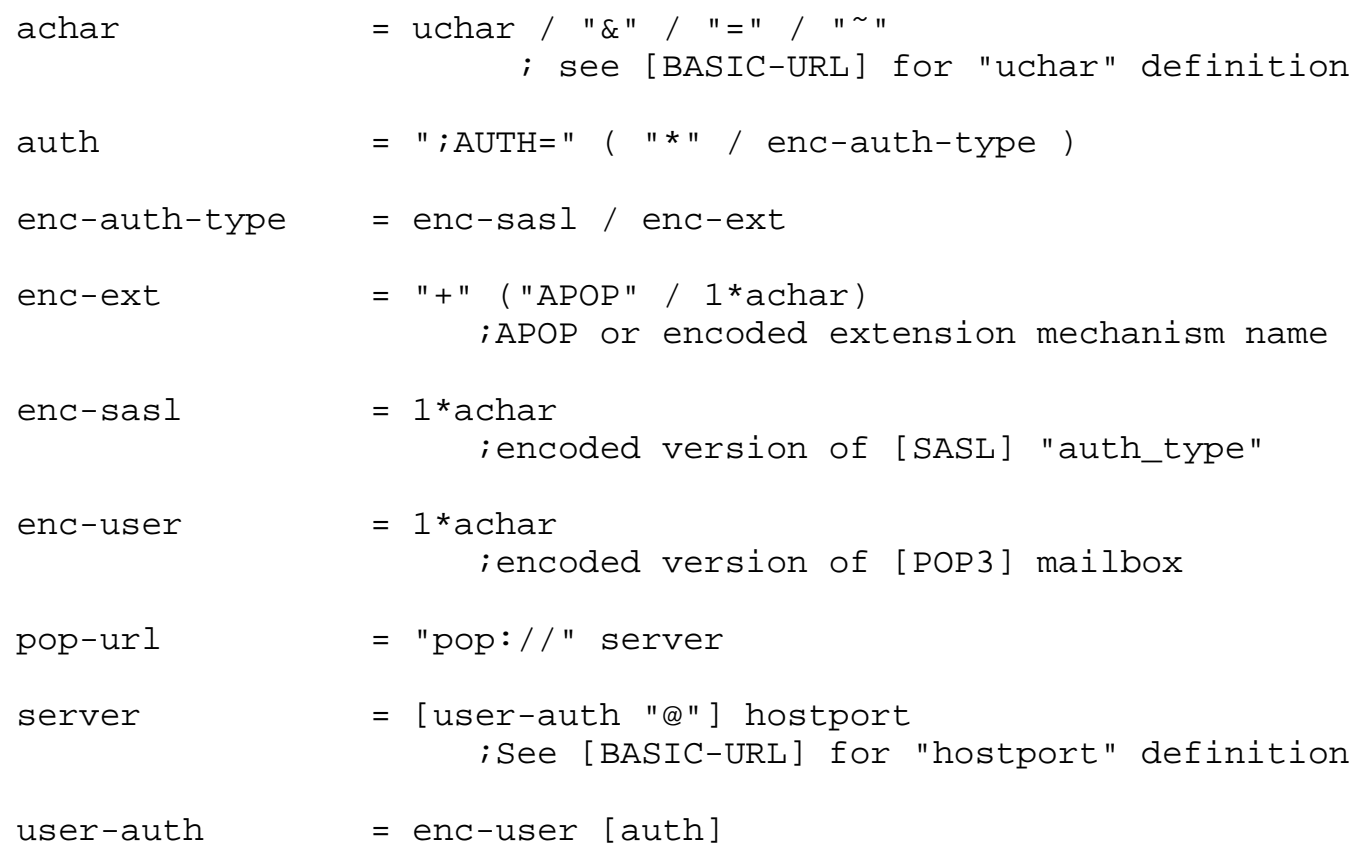

9. Security Considerations

Security considerations discussed in the [POP3] specification and the [BASIC-URL] specification are relevant. Security considerations related to authenticated URLs are discussed in section 4 of this document.

Many email clients store the plain text password for later use after logging into a POP server. Such clients MUST NOT use a stored password in response to a POP URL without explicit permission from the user to supply that password to the specified host name. 


\section{Acknowledgements}

This document borrows heavily from Chris Newman's [IMAP-URL] specification, and has attempted to follow the advice in [URLGUIDELINES ] .

11. References

$[\mathrm{ABNF}]$

Crocker, D., and P. Overell, "Augmented BNF for Syntax Specifications: ABNF", RFC 2234, November 1997 .

$[\mathrm{ACAP}]$

Newman, C., and J. Myers, "ACAP -- Application Configuration Access Protocol", RFC 2244, November 1997 .

[BAS IC-URL]

Berners-Lee, T., Masinter, L., and M. McCahill, "Uniform Resource Locators (URL)", RFC 1738, December 1994 .

[IMAP-URL] Newman, C., "IMAP URL Scheme", RFC 2192, September 1997 .

[IMAP4] Crispin, M., "Internet Message Access Protocol Version 4rev1", RFC 2060, December 1996.

[KEYWORDS] Bradner, S., "Key words for use in RFCs to Indicate Requirement Levels", BCP 14, RFC 2119, March 1997.

[POP-AUTH] Myers, J., "POP3 AUTHentication command", RFC 1734, December 1994 .

[POP3] Myers, J., and M. Rose, "Post Office Protocol -Version 3", STD 53, RFC 1939, May 1996.

[SASL] Myers, J., "Simple Authentication and Security Layer (SASL) ", RFC 2222, October 1997.

[URL-GUIDELINES] Masinter, Alvestrand, Zigmond, "Guidelines for new URL Schemes", Work in Progress.

[UTF8] Yergeau, F., "UTF-8, a transformation format of ISO 10646", RFC 2279, January 1998. 
12. Author's Address

Randall Gellens

QUALCOMM, Incorporated

6455 Lusk Blvd.

San Diego, CA 92121-2779

U.S.A.

Phone: +1 6196515115

Fax: $\quad+16196515334$

EMail: Randy@Qualcomm.Com 
13. Full Copyright statement

Copyright (C) The Internet Society (1998). All Rights Reserved.

This document and translations of it may be copied and furnished to others, and derivative works that comment on or otherwise explain it or assist in its implementation may be prepared, copied, published and distributed, in whole or in part, without restriction of any kind, provided that the above copyright notice and this paragraph are included on all such copies and derivative works. However, this document itself may not be modified in any way, such as by removing the copyright notice or references to the Internet society or other Internet organizations, except as needed for the purpose of developing Internet standards in which case the procedures for copyrights defined in the Internet Standards process must be followed, or as required to translate it into languages other than English.

The limited permissions granted above are perpetual and will not be revoked by the Internet society or its successors or assigns.

This document and the information contained herein is provided on an "AS IS" basis and THE INTERNET SOCIETY AND THE INTERNET ENGINEERING TASK FORCE DISCLAIMS ALL WARRANTIES, EXPRESS OR IMPLIED, INCLUDING BUT NOT LIMITED TO ANY WARRANTY THAT THE USE OF THE INFORMATION HEREIN WILL NOT INFRINGE ANY RIGHTS OR ANY IMPLIED WARRANTIES OF MERCHANTABILITY OR FITNESS FOR A PARTICULAR PURPOSE. 